\title{
Les femmes marginales de l'Histoire : les sorcières de Maryse Condé et de Nancy Huston
}

Maryse SULLIVAN

Université d'Ottawa/University of Ottawa

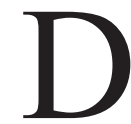

ans cet article, nous analyserons la représentation de la sorcière dans Moi, Tituba sorcière ... de Maryse Condé (1986) et Instruments des ténèbres de Nancy Huston, afin d'observer la construction de cette figure protéiforme et les métamorphoses qu'elle subit. Nous chercherons à mieux comprendre les interactions entre les discours historiques, postcoloniaux et féministes qui ont influencé la composition de la figure de la sorcière dans ces textes. Dans un premier temps, nous étudierons la sorcière dans le roman Moi, Tituba sorcière ... de Maryse Condé (1986); dans un deuxième temps, nous nous pencherons sur le roman Instruments des ténèbres de Nancy Huston paru dix ans plus tard (1996). Dans chaque partie, nous nous intéresserons à la manière dont la sorcière est redéfinie par le texte. Nous serons aussi sensibles aux liens avec l'Histoire établis dans le roman. Les deux livres contiennent d'ailleurs des notes biblio-historiques certifiant que l'intrigue s'inspire de faits réels, créant un dialogue avec le discours historique. Condé trouve son inspiration dans les procès de Salem de 1692 et elle cite même des passages des interrogatoires originaux des accusées de Salem ; Huston, dans un fait divers sur la sorcellerie raconté dans l'étude d'André Alabergère $A u$ temps des laboureurs en Berry (1993). Bien que les sorcières de Condé et Huston aient déjà été abordées de façon séparée, aucune étude n'a jusqu'à présent tenté de rapprocher ces textes qui traitent des enjeux similaires. 
Plus exactement, dans cet article, nous chercherons à voir comment les nouveaux discours historiques, féministes et postcoloniaux s'inscrivent dans les textes de Condé et Huston de manière à redéfinir la marginalité et le statut de la femme en société.

La sorcière et la sorcellerie ont intéressé plusieurs chercheurs d'un point de vue historique, féministe et postcolonial dans les dernières décennies du vingtième siècle. De nombreux historiens (tels Carlo Ginzburg, Robert Mandrou, Robert Muchembled, ou Brian P. Levack) ont interrogé les aspects psychologiques, sociologiques, culturels ou religieux associés à la sorcellerie afin de nuancer, voire transformer, sa représentation comme manifestation historique. ${ }^{1} \mathrm{~S}$ 'intéressant davantage à l'histoire des minorités, des femmes, des paysans, des pauvres, des criminels - en somme des êtres plus marginaux -, ces études sont représentatives de la « nouvelle histoire » qui se concentre sur la vie des individus marginalisés et offre un autre point de vue sur le fonctionnement de la société (Hanciau 123). De la même manière, dès les années 1970, plusieurs études féministes (notamment celles de Catherine Clément et Hélène Cixous, Maroussia Hajduori-Ahmed, Nicole Gnesotto et Lori Saint-Martin²) se sont penchées sur la figure de la sorcière et sur son statut de femme malicieuse et dangereuse. Elles ont réfléchi à la place attribuée à la sorcière et ont réclamé l'égalité des sexes en utilisant cette figure pour faire valoir le pouvoir, la voix et les transgressions de la femme en société ${ }^{3}$ Souvent rattachée à des pratiques traditionnelles caribéennes ou africaines, la figure de la sorcière a aussi été reprise par les études postcoloniales, afin qu'elle représente plus fidèlement les peuples, les coutumes et les discours des différents pays (entre autres dans les travaux de Léonard Sainville, Laënnec Hurbon, Asselin Charles et Honorat Aguessy ${ }^{4}$ ). Plusieurs chercheurs (comme James Haskins, Chadwick Hansen, Robert E. Morsberger et Lloyd W. Brown) se sont également intéressés, dans une perspective postcoloniale, aux figures historiques de descendance africaine ou antillaise qui peuplent l'imaginaire de la sorcière à dessein de se les réapproprier, telle la célèbre Tituba de Salem. ${ }^{5}$

Ce renouvèlement de la figure de la sorcière a trouvé des échos dans des œuvres littéraires contemporaines qui se sont appuyées sur cette représentation " historique » de la sorcière pour en faire une victime en marge de la société. Les romans Moi, Tituba sorcière ... de Maryse Condé et Instruments des ténèbres de Nancy Huston, entre autres, mettent en scène des sorcières, que nous pourrions nommer « historiques », puisqu'ils replacent les personnages dans un contexte historique déterminé. Dans les dernières décennies, divers chercheurs se sont intéressés à la sorcellerie 
d'un point de vue féministe ou postcolonial et ont analysé les œuvres de Condé et Huston. Certaines études ont également exploré la question de l'intertextualité qui ponctue l'œuvre de Condé. Toutefois, aucune analyse n'a rapproché les récits de Condé et Huston pour réfléchir au dialogue qui se construit entre le discours historique sur la sorcellerie et la littérature. En interrogeant les préjugés rattachés à la sorcière, les deux textes font de l'identité et l'histoire des enjeux principaux. Ils redéfinissent les caractéristiques de la figure de la sorcière (son statut, sa perception, sa magie) et transforment certains clichés ou certaines images qui circulent dans l'imaginaire. Ce travail de déconstruction permet alors aux œuvres d'entamer un dialogue avec les discours historiques, postcoloniaux et féministes de la fin du vingtième siècle, qui s'efforcent d'écrire et de mettre en lumière l'histoire des femmes, des Antillais et des marginaux.

\section{Reconstruire l'Histoire en passant par l'histoire}

Moi, Tituba sorcière ... est un roman d'inspiration historique qui, par la narration à la première personne, se veut un récit autobiographique. L'héroïne Tituba raconte « l'histoire de [s]a vie » (Condé 267). Née à la Barbade, où après la mort de sa mère Abena, elle fut initiée aux mystères surnaturels par la guérisseuse Man Yaya, Tituba quitte les Antilles peu après son mariage avec l'esclave John Indien. Leur union l'entraîne à Salem et la projette dans l'atmosphère hystérique puritaine qui donne lieu aux procès de sorcellerie en 1692. Tituba examine son rôle dans les événements, qui ont commencé avec les jeux des filles Betsey et Abigail, et met de l'avant son parcours et sa version des faits.

Le roman s'efforce de mettre de côté l'histoire des sorcières « blanches ", dont le souvenir est demeuré présent dans la mémoire historique officielle, et de s'intéresser au discours et à l'identité d'un personnage souvent oublié et mis à l'écart : la sorcière noire Tituba. Ce choix fait écho aux mouvements postcoloniaux de l'époque qui cherchent à retrouver la singularité des peuples antillais et à retracer leur histoire. Dans les années 1970 et 1980, l'affirmation de l'identité antillaise conduit à une réappropriation du discours historique. Édouard Glissant, entre autres, crée le concept d'antillanité qu'il développe de façon détaillée dans $L e$ Discours antillais (1981) afin d'explorer ce qu'il appelle le «paysage non-possédé » ou " l'anti-Histoire » (152). Celle-ci, qui se veut « des histoires de [leurs] peuples, convergentes », se « débarrasse de la vision linéaire et hiérarchisée d'une Histoire qui courrait son seul fil » (134). Elle ne cherche pas, comme le résume Régis Antoine, à exposer « l'histoire 
officielle évidente, ni une contre histoire univoque de colonisé qui serait symétrique de la première, et aussi peu dialectique[,] [mais] [...] [1]es histoires décalées $[\ldots]$ à haut degré de signification » (361). Relevant moins du discours public enregistré ou de l'hégémonie, ces histoires « reconstituées » montrent les particularités des peuples antillais. En fait, plusieurs des récits de Condé œuvrent à reconstruire l'histoire intercontinentale des diasporas africaines. Ses textes souhaitent tracer leurs origines et leur évolution, de même que retisser les liens coupés entre les cultures (Porter 184 et 196 ; Miller 179; Hewitt 45 ; Araujo 11). En s'intéressant à différents peuples aux Antilles (Traversée de la Mangrove), en Amérique (Moi Tituba sorcière...) et en Afrique (Hérémakhonon), l'auteure explore les interactions entre les Antillais et le monde à travers son œuvre (Hewitt 45 ; Porter 195-96).

Plus précisément, dans Moi, Tituba sorcière..., Condé cultive ces territoires «non-possédés » en mettant en lumière l'histoire personnelle de Tituba. En effet, dans tout le roman, Tituba souhaite que nous écoutions « cette histoire, [s]on histoire » (Condé 156). Même lorsque Hester, l'amie de cellule de Tituba en prison, lui demande de lui réciter un conte, Tituba décide de lui raconter son récit personnel, qui rend compte de ses désirs et de ses malheurs. À ce moment, le roman de Condé montre également comment l'héroïne prend la parole et relate son histoire. Les conteurs et la culture orale sont très importants aux Antilles et le texte rappelle cette tradition en mettant en scène l'énonciation. La formule « Tim tim, bois sèche !» (Condé 156) qu'emploie Tituba pour amorcer son récit est, en fait, le titre d'un conte créole de la Guadeloupe, mais aussi une précaution de départ que le conteur antillais prononce avant de commencer son histoire afin de sonder son auditoire et de savoir s'il est disposé à l'écouter (Bogat). Trop longtemps tenue à l'écart, Tituba choisit donc, non de relater un conte, mais de retracer, à sa manière, son histoire : celle qui l'a entraînée à Salem et a transformé sa vie en " enfer », comme elle l'indique (Condé 157). Ce besoin de prendre la parole et de communiquer son histoire revient souvent dans le roman, ce qui crée un jeu humoristique et métaleptique dans le récit. Tituba avoue notamment ${ }^{6}$

sent[ir] que[,] dans ces procès des sorcières de Salem qui feraient couler tant d'encre, qui exciteraient la curiosité et la pitié de générations futures et apparaîtraient à tous comme le témoignage le plus authentique d'une époque crédule et barbare, [s]on nom ne figurerait que comme celui d'une comparse sans intérêt[,] [celui] [d'] « une esclave originaire des Antilles pratiquant vraisemblablement le hoodoo »[,] [...] [qu'on] ignorerait (Condé 173). 
Le texte inscrit le désir d'énonciation au cœur de l'intrigue pour arracher le personnage à cet oubli. Par la métalepse, il pousse le lecteur à bien considérer la place de Tituba dans l'Histoire et à amincir ou remettre en question les frontières fictionnelles du récit.

En plus d'aborder la question de la place accordée à Tituba dans l'Histoire, le roman de Condé transforme le jugement historique posé sur cette femme. Dans le deuxième chapitre de la partie deux, Tituba prépare, avec l'aide de Hester, la déposition orale qu'elle va faire au tribunal, ce qui a pour effet de modifier le regard porté sur son récit. Souvent considérée comme le catalyseur de l'atmosphère hystérique à Salem - entre autres dans la célèbre pièce américaine The Crucible (1953) d'Arthur Miller -, Tituba affirme dans ces pages que toutes ces histoires de boucs, de chats noirs, de banquet de foetus et de sorcières arrivant sur des balais à des réunions nocturnes lui paraissent « ridicule[s]» (Condé 158). Elle met en doute les termes qui sont employés pour la décrire et réfléchit de manière critique au rôle qu'elle joue en société, comme guérisseuse, conseillère et amie des villageois. Elle demande à Hester "pourquoi [...] dans cette société [occidentale], [on] donn[e] à la fonction de "sorcière" une connotation malfaisante ? " (Condé 152). Selon elle, la sorcière, " si nous devons employer ce mot » (152), devrait être vénérée et inspirer de la gratitude puisqu'elle « corrige, redresse, console, guérit... » (152). Contrairement aux versions antérieures du personnage, la Tituba de Condé rejette les normes et croyances toutes faites lors de son premier contact avec la culture occidentale et à son arrivée aux États-Unis.

En fait, avant de quitter la Barbade, la première maîtresse anglaise de Tituba, Susanna Endicott, l'avait obligée de se convertir au christianisme. L'héroïne possédait donc des connaissances de base du protestantisme et du diable avant son arrivée en Amérique. D'après les recherches d'Artress Bethany White, la conversion d'esclaves aux religions chrétiennes par les Anglais à la Barbade n'était pas commune. Cette réalité est d'ailleurs confirmée par peu de documents archivistiques (148). Condé met toutefois cet aspect de l'avant dans son roman et le présente comme étant d'une importance capitale pour comprendre les relations esclave-maître, et surtout les différentes conceptions de la sorcière. Dans le texte, la religion chrétienne représente l'effort des sociétés européennes ou américaines de « civiliser » leurs esclaves. Cependant, malgré les tentatives de Susanna Endicott et de la famille Parris, Tituba n'adhère pas à leur système de croyances, mais prétend seulement avoir changé ses habitudes. D'un regard détaché, elle interroge alors le statut des « sorcières » et la marginalisation des femmes dans sa nouvelle société. Sans catégoriquement s'opposer 
aux autres et à leur religion, elle continue aussi de pratiquer sa religion originaire, ce qui permet au texte de discuter des traditions antillaises et de nuancer la représentation de la sorcellerie, comme phénomène maléfique et activité vindicative (White 148). En abordant la sorcière de cette façon, le roman confronte des visions du monde antithétiques (antillaise et américaine ; africaine et européenne ; féministe et patriarcale) et dote la sorcière et la femme de qualités positives, en vue de montrer l'ambivalence de cette figure (Wilson 109 ; Jurney 1167 ; Thomas 102).

Par ailleurs, l'œuvre met en relief d'autres dualités et groupes marginalisés. Le personnage de Benjamin Cohen d'Azvedo fait, par exemple, ressortir l'oppression des Juifs à travers l'Histoire. La relation qu'il établit avec Tituba souligne les similarités entre les persécutions des sorcières, des Noirs et des Juifs. ${ }^{7}$ Même si, par moments, Benjamin essaie de prouver à Tituba que son peuple a plus souffert que le sien, il remarque qu'il partage un sort pareil à Tituba (Thomas 90 ; Debrauwere-Miller 224-25). De la même manière qu'elle, il est exclu de la société. Selon Jennifer R. Thomas, le roman tente de déhiérarchiser les différentes identités afin d'exposer, au contraire, leur parcours, de même que leurs particularités communes et individuelles (90-91). Moi, Tituba sorcière ... révèle également l'histoire difficile des Amérindiens, qui ont aussi été persécutés par les peuples blancs (Cotille-Foley 54). Deux Amérindiens racontent à Tituba « comment ils ont été dépossédés de leurs terres, comment les Blancs ont décimé leurs troupeaux et ont répandu parmi eux "l'eau de feu" qui en peu de temps conduit un homme à sa tombe » (Condé 78). Comme les Juifs, les Barbadiens et les Antillais, les Amérindiens ont été dessinés arbitrairement par l'Histoire. En rappelant le parcours de ces peuples et en leur accordant une voix, le roman essaie de montrer à quel point le discours officiel est une construction subjective de l'homme blanc chrétien. La présence, quoique brève, d'autres marginaux et minorités dans le récit élargit alors notre perception de l'Histoire et met en lumière le passé et l'identité de ces groupes, qui peuvent aussi être considérés, en quelque sorte, comme des « sorcières » de l'Histoire.

Par le biais de Tituba, le récit de Condé étend une réflexion sur ces groupes marginalisés, souvent mécompris, et les représente différemment et positivement. En balayant de la main la peur de Satan et les activités qui lui sont rattachées, et en s'interrogeant sur le titre et la place qui sont accordés à la sorcière en société, Tituba déconstruit, par exemple, l'image de la femme naïve, crédule et endiablée, si souvent associée à son personnage. L'héroïne devient un personnage astucieux qui observe, interroge et réfléchit avant d'agir et d'accepter un fait comme une vérité. C'est en 
discutant avec Hester en prison de la place de la femme en société qu'elle comprend ce que signifie son accusation pour sorcellerie. Même si elle considère le tout absurde et exagéré, elle choisit alors de prendre le contrôle de sa situation en préparant, avec l'aide de son amie, un faux témoignage qui lui permettra de protéger ses proches, comme son mari John Indien, et d'éviter la corde et le bûcher. L'attitude trompeuse de Tituba force ainsi le lecteur à remettre en question le document historique intégré au récit : la déposition de l'Antillaise. Le troisième chapitre de la deuxième partie du roman, intitulé « Interrogatoire de Tituba Indien » (163), incorpore d'ailleurs des extraits originaux du témoignage de Tituba se trouvant aux Archives du Comté d'Essex, à Salem, au Massachusetts (voir la note de bas de page dans Condé 165). Cette confession - considérée comme un extrait d'un document juridique véritable - apparaît cependant comme un récit inventé, une performance et un spectacle minutieusement orchestré dans le roman à cause du chapitre qui le précède, qui expose la manipulation. Tituba recourt, entre autres, aux « chats noirs », au « bouc » et au « bec d'aigle » (Condé 157) lors de son témoignage pour répondre aux attentes du tribunal et de l'auditoire et, ultimement, pour sauver sa peau. Dès lors, la survie de Tituba, qui dans l'Histoire et aux yeux des puritains était le résultat de sa déposition honnête, découle désormais d'un jeu et de son habileté à produire adéquatement une parodie de l'identité maléfique qui lui a été imposée à cause de son statut de femme noire (Fulton 50).

En endossant volontairement le stéréotype classique de la sorcière, Tituba transforme la représentation des sorcières de Salem et décompose le récit « officiel » qui lui est associé. La juxtaposition des documents juridiques rédigés par les Blancs et de la narration occultée du personnage marginal permet au roman de créer une fissure dans l'histoire de Tituba. Le texte déconstruit les mythes qui façonnent l'imaginaire d'une communauté et en érige de nouveaux qui sont plus représentatifs de l'histoire du peuple antillais, une fonction qu'Édouard Glissant impute à la littérature antillaise dans les années 1980 (Tamiozzo 138). La redéfinition de la figure de la sorcière, ainsi que la confession douteuse de Tituba poussent alors le lecteur à se poser les questions suivantes : quels autres éléments de son histoire sont méconnus, se peut-il que d'autres parties de son autobiographie soient une fabrication (Peterson 102), ou encore peut-on faire confiance à l'Histoire ?

Dans les faits, l'opposition de l'histoire écrite par les historiens blancs et de l'histoire dissimulée de Tituba modifie aussi les interprétations de sa mort. Avant d'être pendue à la Barbade pour avoir participé à une révolte de Noirs marrons, Tituba entend un planteur blanc crier ses crimes, pré- 
sents et passés, sur l'estrade. À ce moment, elle transmet au lecteur les informations suivantes en employant le pronom « je » pour montrer la façon dont elle est perçue par les troupes anglaises et les autres esclaves dans la foule :

J'avais ensorcelé les habitants d'un village paisible et craignant Dieu. J'avais appelé Satan dans leur sein, les dressant les uns contre les autres, abusés et furieux. J'avais incendié la maison d'un honnête commerçant qui n'avait pas voulu tenir compte de mes crimes et avait payé sa naïveté de la mort de ses enfants. À cet endroit du réquisitoire, je faillis hurler que c'était faux, que c'était menteries, cruelles et viles menteries. Puis je me ravisai. À quoi bon? (Condé 263)

L'emploi du style indirect libre et de la première personne, qui fait écho au « Moi » dans le titre de l'ouvrage, distordent les deux perceptions (celle de Tituba et celle du Blanc) et soulignent, encore une fois, qu'une histoire, et même l'Histoire, peut être fausse. Le texte intègre les pensées de Tituba au compte rendu du planteur blanc pour montrer que, comme à Salem, l'histoire « diffusée » ou " enregistrée » peut être construction erronée. En fait, la tentative de rébellion de Tituba n'est même pas mentionnée dans le discours du planteur, effaçant aux yeux de tous sa mort « noble », pour avoir défendu une cause valide. Les jeux créés dans le texte entre la vérité et l'illusion, de même que le récit personnel et le récit public, forcent le lecteur à porter un regard critique sur les discours qui circulent dans l'imaginaire et à observer les écarts entre ces derniers (Fulton 52). Le roman œuvre à déplacer et à réorienter nos connaissances historiques en vue de faire une place pour la « non histoire ». Le lecteur est alors appelé à reconstruire le discours historique et à le lire autrement (Thomas 88-89). Il est amené à réinterpréter les documents authentiques de l'histoire personnelle de Tituba, à explorer le récit «non officiel » et non documenté de l'accusée et à se réapproprier son histoire afin de lui décerner une vraie identité de femme noire rebelle.

Tout comme Moi, Tituba sorcière..., Instruments des ténèbres de Nancy Huston se charge de ressusciter l'histoire d'une femme accusée de sorcellerie. Par l'entremise de Nadia - personnage-écrivaine dans le récit - , le texte met en scène la relation que l'auteure entretient avec sa création lors de l'écriture. À la manière d'une mise en abyme, il illustre le processus, parfois difficile, de la rédaction d'un roman et les choix auxquels tout auteur doit faire face. Les chapitres d'Instruments des ténèbres alternent 
entre le récit de Nadia au vingtième siècle (intitulé Le carnet Scordatura), qui travaille à la rédaction de son manuscrit, et le récit « écrit » de Barbe Durand (nommé la Sonate de la résurrection), orpheline dès la naissance, qui vécut en France vers la fin du dix-septième siècle. S'inspirant d'un fait divers, de documents authentiques et de l'ouvrage historique $A u$ temps des laboureurs en Berry d'André Alabergère, Nadia retrace l'histoire de la sorcière condamnée à mort en 1712. À l'instar de la vraie Tituba qui n'a pas pu rédiger sa propre autobiographie et dont les seuls souvenirs sont inscrits dans les archives juridiques des procès de Salem (Gyssels 64-65 ; et Thomas 96-97), Barbe Durand n'a pas pu, non plus, écrire son histoire et seules quelques pièces d'archives confirment son existence.

Or, comme Moi, Tituba sorcière..., Instruments des ténèbres ne se limite pas au discours historique enregistré. Nadia souhaite partager le récit de vie de ladite « sorcière » en imaginant son point de vue personnel, de la même manière que le fait Condé dans son roman. L'héroïne-écrivaine de Huston ne s'intéresse pas aux discours des magistrats ou des hommes autoritaires de l'époque qui ont accusée et jugée Barbe, mais à la réalité telle qu'elle se présentait pour une jeune femme orpheline naviguant seule en France à l'époque du Roi-Soleil. Elle met en lumière la vie d'une jeune fille qui « à force d'être immergée tous les six ou huit mois dans une odeur différente, une cacophonie différente, une famille et une basse-cour différentes, à force d'être trimballée, bousculée, caressée et fessée par des mains toujours différentes » (Huston 45) est devenue « le souffre-douleur de tous », car « Barbe Durand n'est pas la fille de personne » (46). Agressée plusieurs fois par son employeur, Barbe devient même enceinte involontairement à l'âge de ses vingt ans. Lorsque vient le temps de rédiger les passages du viol, Nadia avoue éprouver de la difficulté. Néanmoins, elle persévère et demande à son daimôn, l'esprit qui se veut son inspiration et sa muse, « le courage, la vision et la cruauté nécessaire pour conduire cette chose au bout » (Huston 163). Elle sait qu'elle se doit de raconter cette histoire puisque ce sont justement les histoires injustes et pénibles à raconter qui trop souvent demeurent cachées dans l'ombre.

En créant des ponts entre sa propre vie et celle de Barbe, Nadia évoque la vie difficile des femmes à travers les siècles, éclairant les sujets tabous, comme le viol, les difficultés de la maternité et l'avortement : des enjeux qui traversent aussi la critique féministe. Alors que Condé met en lumière la persécution de divers groupes (les marginaux, les femmes, les Noirs, les Juifs, les Amérindiens), Huston se concentre surtout sur l'oppression féminine. Victime du système patriarcal, Barbe est d'ailleurs accusée d'infanticide après avoir donné naissance à un enfant mourant 
non déclaré devant l'Église (Huston 350 et 353). Pourtant, Barbe est avant tout une victime d'agression sexuelle et une pauvre femme qui, à cause de son statut de célibataire, de la malnutrition et de son piètre emploi de servante, ne peut demander de l'aide pendant sa grossesse, ni arracher son bébé à une mort quasi instantanée. Le texte expose la violence faite aux femmes et les injustices qu'elles vivent en montrant l'impuissance de Barbe. Au vingtième siècle, le texte montre aussi comment les femmes sont constamment appelées à sacrifier leur indépendance et leur identité en évoquant l'humiliation et la résignation d'Elisa, la mère de Nadia, forcée à abandonner prématurément sa carrière de musicienne pour servir la famille et remplir son rôle d'épouse et de mère (Arroyas 100). Le roman unit la marginalisation générale des femmes, traitées de sorcières du quinzième au dix-huitième siècle, à l'histoire personnelle de Nadia qui vit la tristesse de sa mère, dut assister à ses nombreuses fausses couches (Huston 37) et vécut, elle-même, des moments difficiles lorsqu'elle choisit de se faire avorter à l'âge de vingt-trois ans. En décrivant la vie de Barbe et en donnant sens à celle-ci, Nadia parvient à exprimer ses sentiments refoulés et à libérer sa haine du passé. L'écriture incarne ainsi, comme le précise Nubia Jacques Hanciau « un exutoire, un dédoublement» (124). Barbe devient le modèle individuel de la «psychose collective » qu'est la sorcellerie, qui peut représenter les persécutions personnelles de toutes les femmes, contemporaines ou des temps passés. Du fragmentaire à la totalité ; du personnel au général ; le roman présente les récits des oubliés de l'Histoire (Hanciau 123-24). Il neutralise le discours officiel afin de privilégier les histoires trop souvent altérées, ignorées ou étouffées des marginaux et des femmes (Sardin 311).

À l'instar du récit de Condé, les deux narrations de Huston mettent également en relief les distinctions existant entre la sorcière guérisseuse et la sorcière diabolique. Alors que la première utilise ses connaissances des plantes pour faire le bien et tient ses secrets de guérison de la gent féminine de la génération précédente qui lui a transmis son savoir avant de mourir - comme le font Man Yaya et Tituba chez Condé -, la deuxième dérive ses pouvoirs de Satan et cherche à faire le mal (Brownlie 73-74) - « des choses étranges et maléfiques » comme l'explique Hester dans le roman de Condé (152). Plus précisément, lorsque Barbe rend service aux membres de la communauté, elle est très bien reçue comme guérisseuse. Cependant, lorsque son enfant ne survit pas après l'accouchement, elle est perçue comme un instrument du diable et une mangeuse d'enfant. De même, quand Nadia décide de mettre un terme à sa grossesse, elle est jugée. Les deux héroïnes apparaissent donc comme des avorteuses : des 
sorcières. En instaurant la distinction entre la sorcière guérisseuse et la sorcière diabolique, le roman de Huston travaille les mêmes enjeux que le texte de Condé, mais y ajoute une dimension contemporaine. Grâce au carnet Scordatura, le récit montre comment la discrimination et l'oppression féminines se poursuivent encore jusqu' au vingtième siècle - et nous pourrions ajouter, au vingt-et-unième siècle - et comment les femmes ne sont pas assez représentées honnêtement dans l'Histoire.

Afin de prendre en charge l'avenir des femmes, Nadia décide alors de transformer la destinée fatale de Barbe Durand. Enflammée d'une fureur féministe et postmoderne, et d'un fervent désir de s'affranchir du joug de son daimôn, elle réécrit l'histoire de la paysanne de manière à lui donner une longue vie et à corriger une injustice. Plus précisément, elle annonce que Barbe, au lieu d'être pendue,

s'acheminera jusqu'à Paris, elle apprendra à lire et à écrire, Louis XIV décèdera et sera remplacé sur le trône par Louis XV, Barbe deviendra célèbre dans la capitale par ses dons en tant que guérisseuse, consolatrice, défaiseuse de sorts, faiseuse d'anges et réparatrice de cœurs brisés ; [...] elle vivra très très vieille, Louis XV décèdera et sera remplacé sur le trône par Louis XVI, Barbe continuera de faire de bonnes affaires comme sorcière, peut-être finira-t-elle par se faire arrêter et enfermer dans l'horrible hôpital général de La Salpêtrière mais, si c'est le cas, elle y fomentera une révolte, poussant les mendiantes, les prostituées, les lépreuses, les syphilitiques et les folles à se soulever : tout est possible, tout est possible, elle n'aura que cent ans en 1786 et elle peut très bien avoir eu un pressentiment de la Révolution française ... (Huston 407-08)

Nadia subvertit l'événement historique, même si ce choix trahit le fait divers authentique dont elle s'inspire, ce qui octroie à l'écriture un pouvoir non seulement créatif, mais aussi transgressif puisqu'il renverse la tradition et les normes établies (Hanciau 125-26). Par la réécriture et les jeux entre le récit-cadre et le récit enchâssé, le roman libère la femme pour lui rendre la parole et lui permettre, en tant que sorcière et être puissant, à intervenir dans l'Histoire.

En plus d'émanciper la sorcière par la réécriture, le texte de Huston laisse la femme s'affranchir en tissant des liens entre la liberté, la folie et la création. Plusieurs des femmes qui peuplent le récit sont écrasées par le système patriarcal comme Elisa, la mère de Nadia, reléguée au foyer 
et forcée de mettre fin à sa carrière de musicienne pour s'occuper des enfants et de son mari abusif ; comme la jeune Jeanne, l'amie de Barbe, obligée de se laisser toucher par les hommes qui habitent l'auberge ; comme Martha, la maîtresse de Barbe, dont la voix, l'opinion et la valeur s'effacent devant son mari et les autorités ; et, bien sûr, comme Barbe qui ne peut utiliser ses talents de guérisseuse ni progresser librement (et sans parents) en société sans être agressée et accusée de tous les maux par la collectivité. Toutefois, après l'accouchement, Barbe arrête de se cacher. Elle n'a plus peur de la vie. Au contraire, elle « a confiance » (Huston 352), " demeure sereine » et « se sent forte, tellement plus forte qu'il y a dix ans, lorsqu'elle était en butte à la haine des habitants de Torchay " (354), qui l'accusaient une première fois. Les villageois s'étant retournés contre elle, " [e]lle est seule» (Michelet 62) comme le dit Jules Michelet dans La sorcière. Désormais, « [e]lle n'a d'ami que ses songes » (Michelet 62), qui prennent la forme de son fils mort prématurément « blotti contre sa peau, comme le Petit Poucet dissimulé dans les habits de la femme de l'ogre » (Huston 352). Elle s'abandonne à une folie paisible et réussit ainsi à se soustraire aux préjugés qui l'emprisonnent lors des interrogatoires. Elle ne cache plus sa grossesse et son passé. L'écrivaine, Nadia, n'accepte pas de mettre en scène un personnage peureux ou dupe. Elle produit une femme complexe qui ne se plie pas aux autorités, « des hommes qu'elle ne connaît pas et qui ne peuvent rien comprendre à ce qu'elle a vécu » (Huston 352). Nadia ne laisse pas l'identité de Barbe être gommée ou sacrifiée pour leur plaire. Même persécutée, cette dernière reste calme, exhibe de la confiance et demeure elle-même.

Le texte nous montre alors deux femmes fortes qui ne se laissent pas amoindrir ou dicter leur rôle par la collectivité ou le système patriarcal. Barbe et Nadia affichent leur marginalité et en sont fières. Elles repoussent les limites de ce qui est considéré comme « acceptable », que ce soit d'un point de vue historique ou social. Selon Hanciau, la folie de Barbe se présente comme le premier pas vers son émancipation et celle de Nadia, sa créatrice, qui s'épanouit à travers elle (125). D'ailleurs, vers la fin du roman, Nadia dit " [s']amuse[r] comme une petite folle » (Huston 403), lorsqu'elle laisse aller les attentes de l'écriture. En se permettant de vivre cette folie, les deux femmes parviennent à libérer leur créativité, jusqu'alors freinée par la société. Cette révolte féminine, qui prend la forme d'une insoumission à la société chez Barbe et une insurrection contre sa muse et l'histoire officielle chez Nadia, est représentative d'une nouvelle entreprise égalitaire et d'une nouvelle liberté créative pour les femmes (Arroyas 99 et Hanciau 125). Barbe et Nadia utilisent et redéfi- 
nissent la marginalité de manière positive. Elles apprennent à embrasser leurs différences, car elles savent maintenant que celles-ci font d'elles des femmes créatives, débrouillardes et stoïques. L'image traditionnelle de la sorcière, qui inclut souvent des femmes laides, veuves, mères-filles ou vieilles, de même que des femmes décrites comme des folles et des hystériques, est transformée. Les sorcières deviennent omnipotentes : capables de résister aux affronts sociétaux, de changer le monde par l'écriture et de faire entendre leur voix. La sorcière est donc reconstruite de manière positive dans le texte afin de permettre à toute femme de surmonter sa situation de mutisme, de crier et de s'affirmer en tant qu'être puissant et important en société. Tout comme le fait Catherine Clément dans son ouvrage important La jeune née paru en 1975, lorsqu'elle indique que la femme libérée, " détachée des regards qui la fixaient en ses crises[,] [...] va, comme la sorcière, s'envoler » (111), le texte de Huston incite les femmes à se réapproprier leur créativité, leur voix, leur langage, leur confiance et à redevenir, pour reprendre les mots de Nadia dans son journal, « ces femmes inspirées dont l'âme était si étonnamment docile qu'il leur suffisait de se frotter le corps d'un onguent et - pouf! - elles s'envolaient par la cheminée sur leur balai » (Huston 32).

\section{Conclusion}

Dans la deuxième moitié du vingtième siècle, de nombreux ouvrages ont analysé la sorcellerie et ses échos en littérature. Cependant, même si plusieurs études se sont attardées au texte de Condé et quelques-unes sur le texte de Huston, aucune n'a pas tenté de rapprocher ses œuvres afin de souligner leur entreprise commune. Les deux textes désirent exposer l'histoire de plusieurs groupes marginaux, dont celles des femmes, en passant par la déconstruction de l'histoire officielle et la construction d'un récit personnel. Plus précisément, les œuvres de Condé et Huston dénoncent les injustices liées au sexe et à la race en cherchant à décomposer les hiérarchies sociales, à se réapproprier l'histoire des femmes et des Antillais et à réparer un tort en accordant enfin une vraie voix au personnage de Tituba et celui de Barbe. Alors que le texte de Condé cherche à faire de Tituba « une héroïne noire dans une Histoire blanche » comme l'explique Florence Ramond Jurney (1161), le récit de Huston cherche à faire de Barbe et Nadia des héroïnes féminines dans une Histoire masculine. Les trois toutefois attaquent les stéréotypes liés aux sorcières et aux femmes. Se situant au carrefour de plusieurs groupes marginaux, leurs voix représentent les voix de multiples minorités étouffées à travers l'Histoire. 
Tituba, Barbe et Nadia nous obligent à repenser l'histoire des femmes, des infortunés, des Noirs, des Antillais, des Amérindiens, des Juifs et des marginaux à dessein de nuancer l'histoire officielle. Les récits de Condé et Huston veulent que nous modifiions les référents historiques qui peuplent notre mémoire collective, c'est-à-dire la mémoire que nous partageons avec les membres de la société qui est parsemée de figures, de traditions et d'événements dont nous avons gardé le souvenir (Halbwachs 103, 113 et 118), pour laisser place à d'autres interprétations. Les femmes des textes réclament leur histoire afin de réécrire l'Histoire, ce qui octroie aux œuvres de Condé et Huston des statuts spéciaux, à la croisée des discours littéraire et historique. Les auteures ne s'adonnent pas seulement à la fiction, mais entreprennent de reconquérir l'Histoire. Quoiqu'au premier abord, leurs buts et leurs récits peuvent sembler bien distincts - les intrigues ont effectivement lieu sur des continents séparés -, Condé et Huston œuvrent toutes les deux à réhabiliter la sorcière en passant par l'histoire et l'Histoire.

La redéfinition de la figure de la sorcière à laquelle participent Condé et Huston mettent également en relief un fil conducteur commun. Moi, Tituba sorcière ... et Instruments des ténèbres accentuent l'ambigüité de la figure de la sorcière, marquée par des connotations à la fois positives et négatives. En réécrivant l'histoire de deux sorcières et en réexaminant leur rôle dans des cas de sorcellerie, les auteures décomposent les récits officiels et les stéréotypes associés à Tituba, l'esclave noire de la Barbade, et aux cas de sorcellerie en général, par le biais de Barbe Durand, la pauvre paysanne française. Elles mettent en scène des femmes complexes (faibles et fortes) qui, malgré les oppressions sociales ou raciales, travaillent et luttent pour transformer leur situation en société. Tituba et Barbe, mais aussi Nadia, se montrent actives : elles affichent de la confiance, acceptent et affectionnent leur corps de femme, résistent à l'assimilation ou à l'ordre établi, et, plus que tout, parviennent à se trouver et à rester fidèle à ellemême même lors de temps difficile.

$\mathrm{Si}$, selon Robert Muchembled, la sorcellerie ne disparaît pas, mais, à travers les siècles, « témoigne encore des résistances [...] [et] porte le souvenir des recettes pour la survie [et] des remèdes contre le désespoir » (254), elle existe aujourd'hui pour servir de nouveaux buts. Elle devient, par sa subsistance dans les textes littéraires, un outil qui permet de résister aux normes établies et de redéfinir la perception de la marginalité. En repensant le passé, Moi, Tituba sorcière ... et Instruments des ténèbres 
résistent au discours officiel et transforment la figure de la sorcière. Condé et Huston illustrent le pouvoir des femmes marginales et, par la réécriture, prennent contrôle de leur histoire.

\section{Notes}

1. Voir, entre autres, les études Les batailles nocturnes: sorcellerie et rituels agraires aux XVI et XVII siècles (1966) et Le sabbat des sorcières (1992) de Carlo Ginzburg sur le sabbat des sorcières; Magistrats et sorciers en France au XVII siècle (1968) et Possession et sorcellerie au XVII ${ }^{e}$ siècle. (1979) de Robert Mandrou sur le fonctionnement judiciaire et le déclin de la sorcellerie ; La sorcière au village (XVe-XVIII siècle) (1979), Les derniers bûchers (1981) et Le roi et la sorcière (1993) de Robert Muchembled sur les causes sociales et culturelles de la sorcellerie; et The Witch-Hunt in Early Modern Europe (1987) de Brian P. Levack pour une chronologie et un survol des variantes géographiques de la sorcellerie en Europe.

2. Plus précisément, voir Catherine Clément et Hélène Cixous. $L a$ jeune née. Union générale d'éditions, 1975 ; Maroussia HajdukowskiAhmed. "La sorcière dans le texte (québécois) au féminin. » French Review, to 58.2 (1984), 260-68 ; Nicole Gnesotto. « L'imaginaire féministe. » Esprit, to 33/34 (1979), 152-61 ; et Lori Saint-Martin, « Écriture et combat féministe : Figures de la sorcière dans l'écriture des femmes au Québec. »Quebec Studies, 12 (1991), 67-82.

3. Une revue féministe nommée Sorcières voit même le jour en France en 1976 sous la direction de Xavière Gauthier (Goldblum).

4. Plus précisément, voir Léonard Sainville. «Les fondements négroafricains de la culture dans les Caraïbes et la lutte pour leur sauvegarde. » Présence Africaine, 101/102 (1977), 129-57 ; Laënnec Hurbon. « Sorcellerie et pouvoir en Haïti. » Archives de sciences sociales des religions, no 48.1, 1979, p. 43-52 ; Asselin Charles. « Voodoo Myths in Haitian Literature. » Comparative Literature Studies, vol. 47, no 4, déc. 1980 ; et Honorat Aguessy. «Les dimensions spirituelles : religions traditionnelles africaines. » Présence Africaine, no 117/118, 1981, p. 138-48.

5. Voir, par exemple, les études sur Tituba de James Haskins. Witchcraft, Mysticism and Magic in the Black World. Garden City : Doubleday, 1974 ; de Chadwick Hansen. " The Metamorphosis of Tituba, or Why American Intellectuals Can't Tell an Indian Witch from a Negro. » The New England Quaterly, vol. 47, no 1, mar. 1974, p. 3-12 ; de Robert E. Morberger. «The Further Transformation of Tituba. » The New England 
Quarterly, vol. 47, no 3, sep. 1974, p. 456-58 ; ou de Lloyd W. Brown. « Tituba of Barbados and the American Conscience: Historical Perspectives in Arthur Miller and Ann Petry. » Carribean Studies, vol. 13, no 4, jan. 1974, p. 118-26.

6. La métalepse signale une rupture dans l'univers du récit, ce qui pousse le lecteur et parfois les personnages à remettre en question les frontières fictionnelles. Pour plus d'amples renseignements, voir Gérard Genette, Métalepse : De la figure à la fiction, Paris : Seuil, 2004.

7. Il faut noter que d'autres ouvrages littéraires et historiques avaient déjà établis des liens entre la persécution des sorcières et des Juifs. Voir, notamment, Joshua Trachtenberg, The Devil and the Jews, New Haven : Yale University Press, 1943 et Marion L. Starkey, The Devil in Massachussetts. New York : Knopf, 1949.

\section{Bibliographie}

Aguessy, Honorat. «Les dimensions spirituelles : religions traditionnelles africaines ». Présence Africaine, no 117/118, 1981, pp. 138-48.

Antoine, Régis. La littérature franco-antillaise. Paris : Karthala, 1992.

Araujo, Nara. «La raison du mouvement : Hommage à Maryse Condé.

» L'œuvre de Maryse Condé. Dirigé par Nara Araujo, Paris : L'Harmattan, 1996, pp. 9-20.

Arroyas, Frédérique A. Y. «Diabolus in musica : "La Sonate de la Résurrection" de Heinrich Biber, arme de détournement dans Instruments des ténèbres de Nancy Huston. " L'Esprit Créateur, vol. 47, no 2, été 2007, pp. 88-100.

Bogat, Roland. «Tim Tim Bois Sec. » Revue Guadeloupéenne, nouvelle série, no 15, jan-fév. 1948, http://www.potomitan.info/atelier/contes/ conte_creole29.php

Brown, Lloyd W. « Tituba of Barbados and the American Conscience: Historical Perspectives in Arthur Miller and Ann Petry. » Carribean Studies, 13.4 (1974), 118-26.

Brownlie, Siobhan. "Translation and the Fantastic: Nancy Huston's Instruments des ténèbres. » French Forum, vol. 34, no 1, hiver 2009, pp. 67-83.

Charles, Asselin. « Voodoo Myths in Haitian Literature. » Comparative Literature Studies, vol. 47, no 4, déc. 1980, pp. 391-98.

Clément, Catherine, et Hélène Cixous. La jeune née. Paris : Union générale d'éditions, 1975.

Condé, Maryse. Moi, Tituba sorcière ... Paris : Mercure de France, 1986. 
Cotille-Foley, Nora. « Epistémê esclavagiste et sorcellerie subalterne de Loudun à Salem, en passant par Jules Michelet et Maryse Condé. » Nouvelles Études Francophones, vol. 25, no 1, printemps 2010, pp. 46-58.

Debrauwere-Miller, Nathalie. «Au carrefour de la négritude et du judaïsme : Moi, Tituba sorcière ... noire de Salem. » Romantic Review, vol. 90, no 2, 1999, pp. 223-33.

Fulton, Dawn. Signs of Dissent: Maryse Condé and postcolonial criticism. Charlottesville : University of Virginia Press, 2008.

Genette, Gérard. Métalepse : De la figure à la fiction. Seuil, 2004.

Ginzburg, Carlo. Les batailles nocturnes : sorcellerie et rituels agraires aux $X V I^{e}$ et $X V I I^{e}$ siècles. Traduit par Giordana Charuty. Paris : Flammarion, 2010.

Ginzburg, Carlo. Le sabbat des sorcières. Traduit par Monique Aymard. Paris : Gallimard, 1992.

Glissant, Édouard. Le discours antillais. Paris : Seuil, 1981.

Gnesotto, Nicole. " L'imaginaire féministe. » Esprit, no 33/34 (9/10), sep-oct. 1979, pp. 152-61.

Goldblum, Caroline. «Sorcières, 1976-1981. Étude d'une revue féministe. Master 1, Université de Lille III, (dir. Florence Tamagne), 2009. » Genre et Histoire, no 8, Printemps 2011, http://genrehistoire.revues. org/1217.

Gyssels, Kathleen. « On the Untranslatability of Tituba Indian, An Intercultural Subject. » Traduit par Victoria Bridges Moussaron. Emerging Perspectives on Maryse Condé : A Writer of Her Own. Dirigé par Sarah Barbour et Gerise Herndon. Trenton : Africa World Press, 2006, pp. 63-86.

Hajdukowski-Ahmed, Maroussia. « La sorcière dans le texte (québécois) au féminin. » The French Review, vol. 58, no 2, déc. 1984, pp. 260-68.

Halbwachs, Maurice. «La mémoire collective et le temps. » La mémoire collective. Paris : Presses universitaires de France, 1950.

Hanciau, Nubia Jacques. « La sorcière chez Nancy Huston : de l'ancien au nouveau monde. »Interfaces Brasil/Canada : Porto Alegre, vol. 1, no 2, 2002, pp. 119-28.

Hansen, Chadwick. « The Metamorphosis of Tituba, or Why American Intellectuals Can't Tell an Indian Witch from a Negro. » The New England Quaterly, vol. 47, no 1, mar. 1974, pp. 3-12.

Haskins, James. Witchcraft, Mysticism and Magic in the Black World. New York : Doubleday, 1974. 
Hewitt, Leah. " Recontres explosives : les intersections culturelles de Maryse Condé. » L'œuvre de Maryse Condé. Dirigé par Nara Araujo. Paris : L'Harmattan, 1996, pp. 45-56.

Hurbon, Laënnec. « Sorcellerie et pouvoir en Haïti. » Archives de sciences sociales des religions, no 48.1, 1979, pp. 43-52.

Huston, Nancy. Instruments des ténèbres. Actes Sud/Léméac, 1996.

Jurney, Florence Ramond. « Voix sexualisée au féminin dans "Moi, Tituba sorcière" de Maryse Condé. » The French Review, vol. 76, no 6, mai 2003, pp. 1161-71.

Levack, Brian P. The Witch-Hunt in Early Modern Europe. Longman, 2016. Mandrou, Robert. Possession et sorcellerie au XVII siècle. Paris : Fayard, 2005.

Mandrou, Robert. Magistrats et sorciers en France au XVII ${ }^{e}$ siècle. Paris : Seuil, 1980.

Michelet, Jules. La Sorcière. 1862. Paris : Garnier-Flammarion, 1966.

Miller, Christopher. «After Negation: Africa in Two Novels by Maryse Condé. » Postcolonial Subjects: Francophone Women Writers. Dirigé par Mary Jean Green et al., Minneapolis : University of Minnesota Press, 1996, pp. 173-85.

Morberger, Robert E. « The Further Transformation of Tituba. » The New England Quarterly, vol. 47, no 3, sep. 1974, pp. 456-58.

Muchembled, Robert. Le roi et la sorcière. Paris : Desclée, 1993.

Muchembled, Robert. La sorcière au village (XV'-XVIII siècle). Paris : Gallimard-Julliard, 1991.

Muchembled, Robert. Les derniers bûchers. Paris : Ramsay, 1981.

Peterson, Carla. « Le surnaturel dans Moi, Tituba sorcière ... Noire de Salem de Maryse Condé et Beloved de Toni Morrison. " L'œuvre de Maryse Condé. Dirigé par Nara Araujo. Paris : L'Harmattan, 1996, pp. 91-104.

Porter, Laurence M. « Maryse Condé, Historian of the Black Diaspora. " Emerging Perspectives on Maryse Condé, A Writer of Her Own. Dirigé par Sarah Barbour et Gerise Herndon. Trenton : Africa World Press, 2006, pp. 181-99.

Saint-Martin, Lori. «Écriture et combat féministe : Figures de la sorcière dans l'écriture des femmes au Québec. "Quebec Studies, vol. 12, printemps-été 1991, pp. 67-82.

Sainville, Léonard. " Les fondements négro-africains de la culture dans les Caraïbes et la lutte pour leur sauvegarde. » Présence Africaine, no 101/102, 1977, pp. 129-57. 
Sardin, Pascale. «Towards an Ethics of Witness, or the Story and History of "une minuscule détresse" in Annie Ernaux's L'Événement and Nancy Huston's Instruments des ténèbres. » French Studies : A Quarterly Review, vol. LXII, no 3, 2008, pp. 301-12. ProQuest, doi : http:// dx.doi.org.proxy.bib.uottawa.ca/10.1093/fs/knn027.

Tamiozzo, Josée. «L'altérité et l'identité dans Moi, Tituba sorcière... noire de Salem, de Maryse Condé. » Recherches féministes, vol. 15, no 2, 2002, pp. 123-40.

Thomas, Jennifer R. « Talking the Cross-Talk of Histories in Maryse Condé's I Tituba, Black Witch of Salem. » Emerging Perspectives on Maryse Condé : A Writer of Her Own. Dirigé par Sarah Barbour et Gerise Herndon. Trenton : Africa World Press, 2006, pp. 87-104.

White, Artress Bethany. « From Africa to America by Way of the Caribbean: Fictionalized Histories of the African Diasporic Slave Woman's Presence in America in I, Tituba, Black Witch of Salem and A Mercy ». Literary Expressions of African Spirituality. Dirigé par Carol P. Marsh-Lockett et Elizabeth J. West. Lanham : Lexington Books, 2013, pp. 145-60.

Wilson, Elizabeth. " Sorcières, sorcières : "Moi, Tituba sorcière... Noire de Salem", révision et interrogation. " L'ouvre de Maryse Condé. Dirigé par Nara Araujo. Paris : L'Harmattan, 1996, pp. 104-13. 